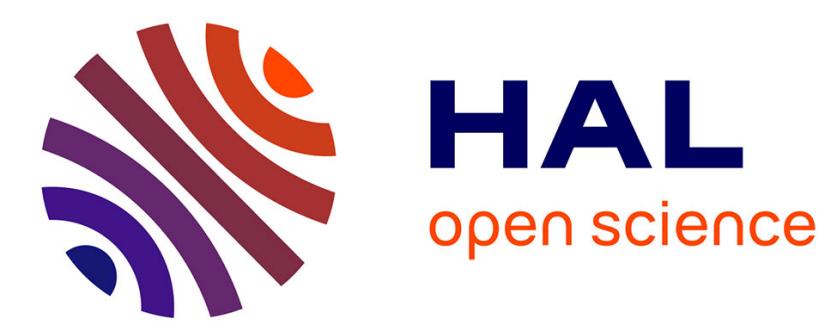

\title{
A design space of guidance techniques for large and dense physical environments
}

Hind Gacem, Gilles Bailly, James R Eagan, Eric Lecolinet

\section{To cite this version:}

Hind Gacem, Gilles Bailly, James R Eagan, Eric Lecolinet. A design space of guidance techniques for large and dense physical environments. IHM'14:Proceedings of the 26th Conference on l'Interaction Homme-Machine, Oct 2014, Villeneuve d'Ascq, France. pp.9-17, 10.1145/2670444.2670455 . hal01147720

\section{HAL Id: hal-01147720 https://hal-imt.archives-ouvertes.fr/hal-01147720}

Submitted on 6 May 2015

HAL is a multi-disciplinary open access archive for the deposit and dissemination of scientific research documents, whether they are published or not. The documents may come from teaching and research institutions in France or abroad, or from public or private research centers.
L'archive ouverte pluridisciplinaire HAL, est destinée au dépôt et à la diffusion de documents scientifiques de niveau recherche, publiés ou non, émanant des établissements d'enseignement et de recherche français ou étrangers, des laboratoires publics ou privés. 


\title{
A design space of guidance techniques for large and dense physical environments
}

\author{
Hind Gacem ${ }^{1,2} \quad$ Gilles Bailly $^{2,1} \quad$ James Eagan $^{1,2} \quad$ Eric Lecolinet $^{1,2}$ \\ ${ }^{1}$ Télécom ParisTech, ${ }^{2}$ CNRS LTCI UMR 5141, Paris, France \\ \{firstname\}.\{lastname\}@telecom-paristech.fr
}

\section{ABSTRACT}

Finding an object in a physical environment is difficult if the environment contains many objects, especially if it is large and dense. We propose a design space that describes and compares existing guidance techniques according to four dimensions: output modality, physicality, granularity and spatial information. Output modality can be visual, audio or tactile. Guidance information can be displayed using physical objects or virtual artifacts. Granularity indicates whether the technique serves to navigate towards the vicinity of the target or to precisely localize the target. Finally, spatial information is either exocentric or egocentric. This design space aims at providing an overview of the domain and helping designers and researchers to understand the key properties of these techniques. It also enables their comparison and the generation of new techniques by highlighting unexplored areas.

\footnotetext{
Mots Clés

Visual search; Navigation; Guidance

Techniques; Design space;
}

\section{ACM Classification Keywords}

H.5.m. Information interfaces and presentation (e.g., HCI): Miscellaneous.

\section{INTRODUCTION}

Locating an object in a physical place is a common activity in the real world. Such a task can quickly become difficult if the environment contains many objects, especially if it is large and dense. For instance, libraries, supermarkets, factory plants, etc., can contain thousands of objects with many of them very close to each other. Moreover the size of these environments makes the problem harder [1] as the user must not only find the desired object in the proper area, but also find where this area is located in the environment.

Various guidance techniques have been proposed in the literature (e.g. [24, 13, 48]) for finding objects in such environments. However, the literature does not systematically describe (1) the differences and similarities of these techniques, (2) their relative advantages and drawbacks, and (3) their interactions with the different output modalities and the complexity of the environment.

In this paper, we propose a design space to describe and compare existing guidance techniques for indoor search tasks in a large and dense environment. This design space is organized according to four dimensions. The first dimension corresponds to the output modality used by the technique. In addition to the traditional visual, audio and tactile categories, we also attempt to take into account the impact of physicality through a second dimension, which corresponds to the case when guiding information is embedded into physical objects.

The third dimension of our design space is the granularity of the technique, which can either provide micro or macro guidance. This axis is important because it corresponds to two different kinds of tasks and user behaviors and thus to different classes of techniques that do not require the same level of precision and 
resolution. Moreover, this dimension also helps understand how these techniques can be combined to build hybrid techniques that provide both macro and micro guidance. Finally, the fourth dimension indicates the type of spatial information that is provided by the technique, which can be either exocentric or egocentric.

Previous explorations of such guidance techniques often treated these axes independently, especially for what concerns granularity. Our key insight is that many search tasks involve both the macro and micro phases and should thus provide hybrid guidance techniques that combine different modalities and spatial relationships depending on the local granularity.

In this paper we present a design space that is capable of reflecting these hybrid guidance techniques. More specifically, the aims of this paper are 1) to provide an overview of the field of guidance techniques; 2) to help designers and researchers understand the key properties of these techniques, compare them and choose the one that fits their needs; 3) to invite researchers to investigate unexplored areas and to generate new, possibly hybrid, techniques.

\section{DESIGN SPACE}

Guidance techniques help the user to find, locate, and reach a target without having to perform an exhaustive search within the space. They generally have two characteristics: 1) they orient the user's body movement, perhaps iteratively, to reach the target location, and 2) they indicate the target location.

These orientations and indications may use various modalities to present information, such as visually, using sound, etc. They may be embedded in physical objects or use virtual hints. They may operate at different granularities depending on whether the target in the user's field of view or on the size of the target. Finally, the technique may provide these cues using exocentric or egocentric spatial relationships. Together, these four axes make up the dimensions of our proposed design space, which we describe below.
We further provide two representations of this space, depicted in Figures 1 and 2. The first representation is modality-centric and is especially useful for identifying comparable guidance techniques. The second is granularityoriented and is better suited to the hybrid guidance techniques that combine different techniques for different granularities.

In the remainder of this section, we introduce these dimensions and related work. In the next sections, we describe these dimensions in more detail, and explore two representations of this design space.

Modality. The modality is the sensory modality [35]. It is used to convey its guidance hints to the user. Of the five human senses (sight, sound, touch, smell, and taste), we didn't include the last two, in the design space representation, because techniques based on olfactory and gustatory interaction are still in an early stage of development [27] and hence seldom used in augmented reality systems [55].

Physicality. Beyond these base modalities, physical objects have presence, which may be perceived via any of these senses or a combination of them. Physical objects provide these modalities in a consistent manner and with a higher level of realism than virtual artifacts. For instance, Danieau showed that combining virtual modalities (audio, visual and tactile) enhances perception but still does not provide the same feeling of realism [7]. Moreover, physical objects do not require users to wear AR devices (headphones, headmounted displays, force-feedback exoskeletons, etc.) that may be intrusive or cumbersome and may limit users.

Granularity. Indoor search tasks consist of two primary subtasks: macro navigation and micro navigation $[22,50]$. Macro navigation generally corresponds to the coarse phase of locating the vicinity of the target, such as the shelf on which a book resides. Micro navigation involves the finer-grained location of an object already within the field of view, such as finding a jar of jam when staring at an open refrigerator. 


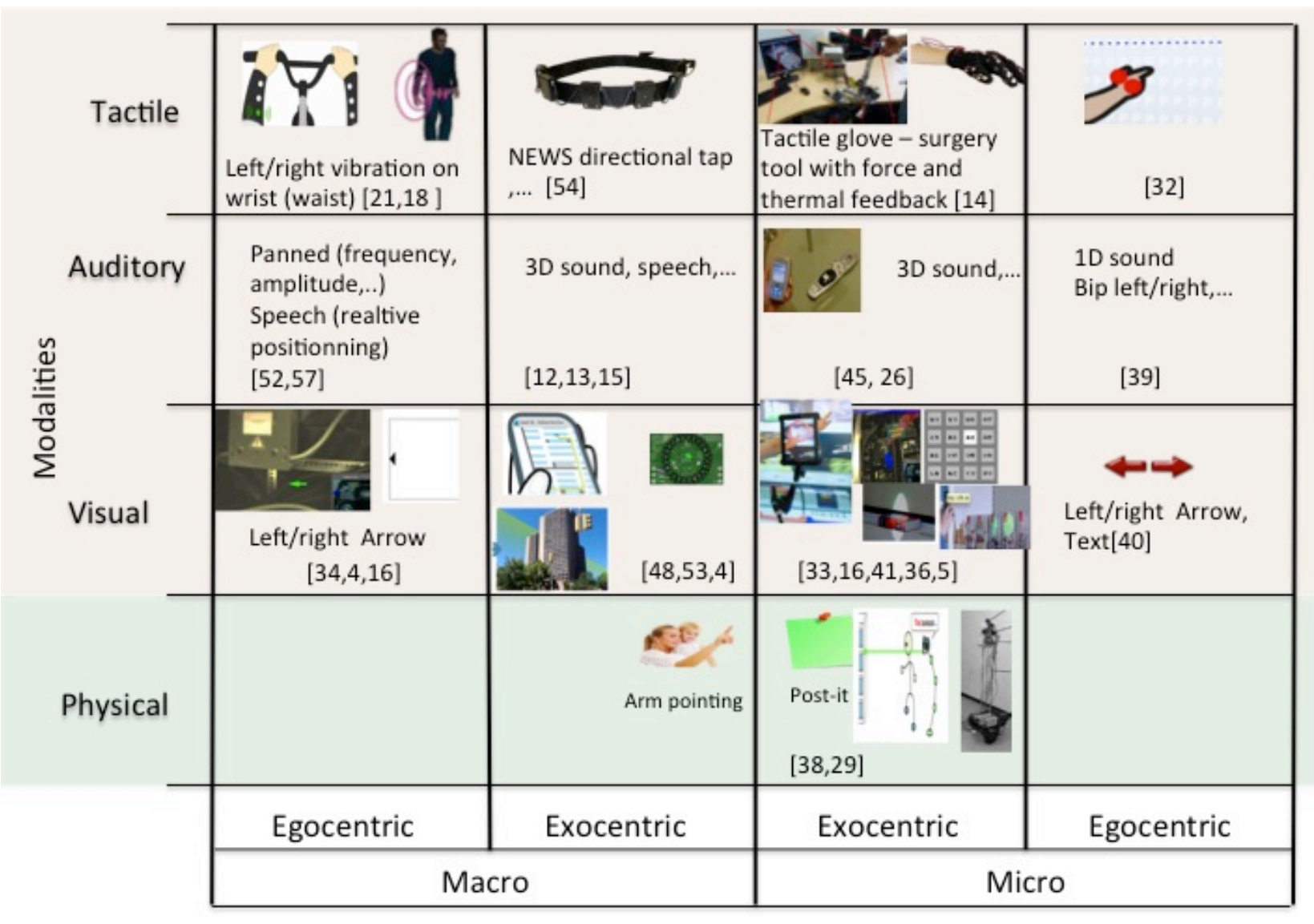

Type of guidance technique (Macro/Micro, Exocentric/Egocentric)

Figure 1: First representation of the design space, which classifies guidance techniques according to modality, physicality, granularity (macro/micro) and the spatial guidance type (egocentric/exocentric). Physicality and modalities are two orthogonal dimensions represented on the same axis to simplify the visualization.

Spatial Guidance Type. The spatial guidance type describes the frame of reference in which the system provides guidance, which may be exocentric or egocentric. Exocentric guidance provides information related directly to a position or a direction in the environment, be it the user, the environment, or the target. Egocentric guidance, on the other hand, provides only a partial guidance, relative to the position and orientation of the user, such as by a vibration on a wrist strap indicating to turn right [21]. In this latter case, the guiding information is generally provided iteratively to tell the user in which direction he should go or look for the target.

\section{Related Work}

Many studies propose guidance techniques to localize virtual targets in virtual environments (e.g. $[37,8]$ ), we focus on the guidance and localization of real objects in the real world.

Several studies have attempted to characterize various guidance techniques. We are not aware, however, of other design spaces of guidance techniques. Buchmann et al. [4] have characterized guidance techniques according to the type of information. They distinguish between angular (compass) and non-angular information (left/right arrow) and between onbody (direct) and off-body (indirect, on external devices) techniques. The first of these types of information fits well within our definition of the spatial guidance type. Furthermore, Buchmann et al. focus on macro guidance techniques and do not take into account micro ones. For instance, their classification does not adequately account for 
the Shelftorchlight [36], which uses micro navigation guidance.

Guidance techniques typically rely heavily on indoor localization, for which many characterizations exist (see $[47,49]$ for a literature review). However, these works focus more on algorithmic and technological aspects rather than on the higher-level interaction and guidance.

We now present several guidance techniques to illustrate our design space. They are organized according to our four axes.

\section{DESIGN SPACE DESCRIPTION}

In constructing this design space, we aimed to take a person-centric approach. The user and his or her goal of locating an object guide the design of the four dimensions: the user's senses inform the modality; the way of displaying information informs the physicality; the relationship between the user and the target informs the granularity; and the frame of reference between the two informs the spatial guidance type.

We purposely avoid directly considering technology among these axes. Different tracking systems have different characteristics of precision, accuracy, and scale, and thus limit which guidance techniques can be used with which techniques. Different presentation technologies such as head-mounted displays, projectors, vibro-tactile wristbands, etc., further influence which modalities, granularities or spatial guidance types are appropriate.

Together, these four dimensions serve to provide an abstract overview of augmented reality guidance techniques. In the remainder of this section, we consider these dimensions and influences in more detail.

\section{Modality}

Each of the five human senses offers different properties that make it more or less suitable for different kinds of tasks [13]. For example, vision offers a higher information bandwidth than audio [20], but audio can remain effective when a target is beyond the field of view.
Visual. Visual guidance techniques are perhaps the most common of the modalities. They may use text [40,51] light [36] or shape [33] in 2D or 3D space [16]. Furthermore, displayed information may be static or animated.

Audio. Auditory guidance techniques map sound parameters [17] such as frequency [57], amplitude and rhythm [2], or use auditory icons $[11,17]$, earcons [42,17], vowel sound mapping [15], spatialization through 3D sound [30,52], or combinations of sonification and spatialization $[37,12]$ to convey guidance information. They may alternatively use speech synthesis to provide verbal guidance information. Using 3D spatial mapping techniques, in headphones [30] or in speaker networks [45], can provide a rich, natural spatial mapping [12]. Alternatively, simple stereo headphones can provide for more basic left/right guidance relative to the user.

Tactile. Tactile guidance techniques use the tactile feedback perceived by the human body. Tactile feedback may take several forms: attractive or repulsive forces [37,31], vibrations $[46,44]$, temperature $[23,10]$, pressure [19], electric current $[25,28,43]$ or a combination of these parameters $[14,58]$. Tactile guidance may rely on simple feedback or sequential patterns $[18,32]$ and can take place anywhere on the body (belt [18], wrist [21], back [44,54]...). On this subject, Cholewiak et al. suggest that anatomical reference points are more effective for tactile feedback perception [6].

\section{Physicality}

Physicality is an orthogonal dimension in regard to modality. Physical objects intrinsically exist in 3D space and can be directly perceived through the visual, audio and tactile modalities. Nonetheless, the fact that an object is physical can alter the nature of the object to such a degree that we consider its physicality to be paramount to the particular senses involved.

Physicality may facilitate the interpretation and understanding of information conveyed using virtual representations of the same modalities. 


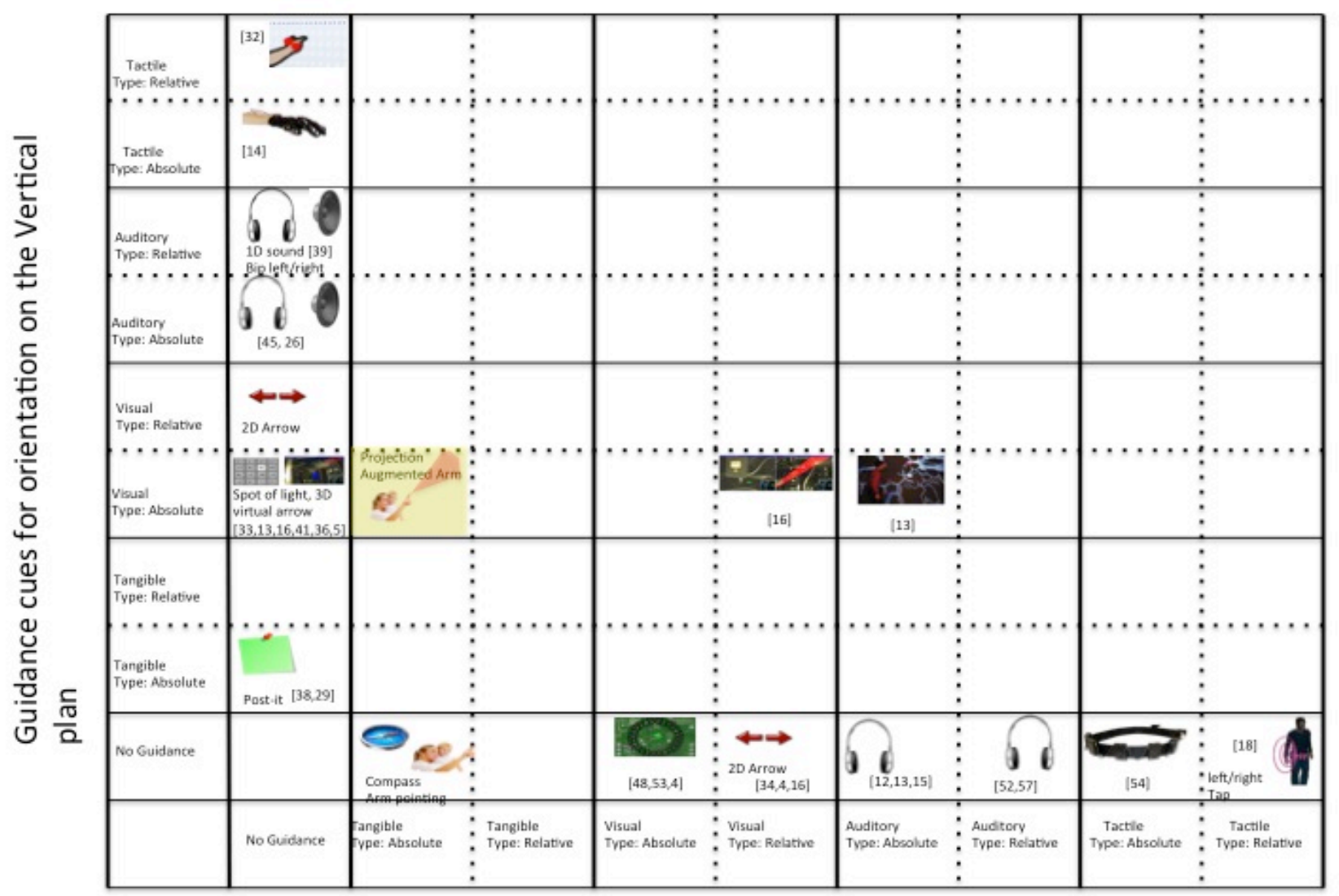

Guidance cues for orientation on the horizontal plan

Figure 2: The second representation of the design space, which respectively displays micro and macro guidance techniques on the vertical and horizontal axes. The "no guidance" column corresponds to micro-guidance techniques that provide no macro-guidance (and vice-versa for the "no guidance" row). Most techniques tend to focus on either macro (bottom row) or micro (left axis) navigation. Techniques in the middle combine micro and macro granularities. The table highlights that many combinations have not yet been explored so far.

For example, Kuzuoka et al. observe that the physical presence of a robot instructor makes the user aware of its movement and that the user could use this information to predict the location of the target pointed by the robot [29]. In another study, Mikawa et al. found that using a physical object (the head of a robot) to guide a library user would help him to understand guidance information [38]. Finally, $\mathrm{Ju}$ and Sirkin have shown that using a physical arm could be effective to attract the user's attention [56].

Physical visual guidance use movement, shape and the state of physical objects to specify a target as in Kuzuoka et al.'s work [29]. In contrast, virtual visual guidance means that synthetized representations are used, as in [16] where a 3D arrow pointing to a target is displayed on a head mounted display.
Physical auditory guidance, which is emitted directly by physical objects [26], provides information on their 3D location and changes of state (e.g. the object is moving, stopped, collided...). Virtual auditory guidance relies on computer generated sounds that are emitted virtually in real space. Virtual sound can be played on headphones using head-related transfer functions $[30,12]$ or by loudspeakers [45].

Physical tactile guidance results from touching physical objects and combines information on shape, changes in shape, acceleration, vibrations and temperature. Virtual tactile guidance requires creating illusions to provide feedback during interaction with virtual objects by using a cross-modal effect or by simulating tactile feedback using electrical [25,43], thermal or force [14]. For example, Ban et al. 
propose a cross-model effect (combining visual and static tactile feedback) to create the illusion of tactile perception of the shape of virtual objects [3]. An example of simulated tactile feedback is the display proposed by Kajimoto et al. which simulates uses electrical stimulation to simulate tactile feedback (the sensation of pressure and vibration) [25].

In order to simplify the representation of the design space, Figures 1 and 2 project modality and physicality onto a single axis.

\section{Granularity}

Search tasks involve various phases depending on the scale of the target and its distance from the user. Generally, this task can be broken down into two broad types: macro navigation and micro navigation $[22,50]$.

In macro navigation, the user tends to perform large movements, re-orienting him- or herself and moving around within the environment [54,4,21]. In micro navigation, the user is generally in the proximity of the target and performs a finer grained search, such as by scanning the field of view with the eyes [41, 16], hands [14] or both [32].

A guidance task that is well suited to macro navigation may not be particularly well-suited to micro navigation and vice versa. For example, drawing a small circle around a target may be effective when near the target $[41,39]$, but if that target is on the other side of the room, it may be too small to identify. Likewise, emitting a beep from a small switch may be sufficient to lead the user across the room to a switchboard, but may be insufficient to identify the particular switch.

\section{Spatial Guidance Type}

Exocentric. These techniques display information in fixed locations or toward a fixed direction in space. As such, when the user moves or turns his or her body, the guidance information continues to present the same information. Examples of this type of technique include a mini-map in a videogame, a map [34, 24] (Figure 3c); a spotlight on the target $[5,41]$ (Figure 3b); modifying the target [53] or a beep emitted from the target [26].
Egocentric. These techniques display information that is directly related to the user's location in the physical space. The information may be a sequence of instructions that the user needs to follow iteratively. Examples include projected arrows pointing to the left and to the right [34] (Figure 3a); text [40]; left/right beeps on headphones [57] or loudspeakers [39]; left/right tap on tactile belt [18], or a tap pattern with tactile feedback [9].

\section{DISCUSSION}

We provide two primary representations of the design space, as shown in Figures 1 and 2. Both of these representations provide a twodimensional visualization of the proposed fourdimensional design space. Figure 1 shows modality and physicality vs. granularity and spatial representation type. Figure 2 focuses on the granularity of the guidance technique.

Orienting the second representation around the granularity of the guidance technique makes it easier to compare hybrid guidance techniques. These techniques are often used to provide one kind of guidance for the macro phase of the search task and another for the micro phase of the search task, as in Grohn et al. [13], who use 3D exocentric audio for coarse guidance, reinforced by a spotlight on the target (absolute visual guidance) for fine-grained guidance.

Together, these representations help show the descriptive, comparative, and generative capabilities of this design space.

Descriptive power. The proposed design space breaks guidance techniques down across the modality used to guide the user, the relationship between the user and the target, and the granularity of the search task.

This breakdown makes the proposed design space particularly good at describing hybrid guidance techniques, especially when using the second representation, as shown in Figure 2.

The majority of guidance techniques tend to focus on either macro or micro navigation. For example, all the techniques along the bottom row of Figure 2 provide macro guidance but 


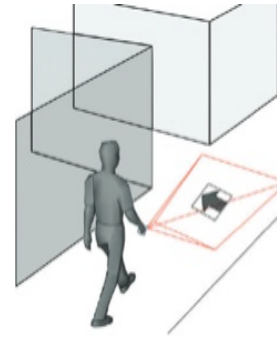

(a)

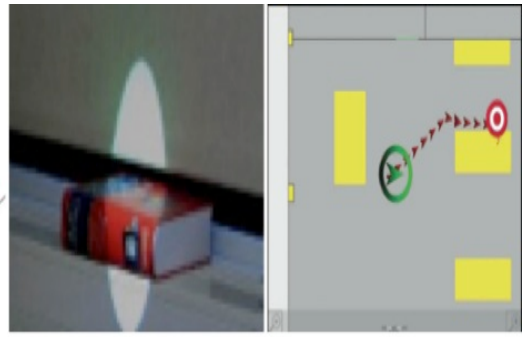

(b)

(c)
Figure 3 (a): Projected left/right arrow [34]. (b): A spotlight on the target [5]. (c): A map with the path [24].

no support for fine-grained micro navigation.

Along the left axis, techniques provide micronavigation support, but do not target coarser macro-navigation. Techniques in the middle, however, combine modalities to support both granularities, enabling a broad description of even hybrid guidance techniques.

For example, Löchtefeld et al.'s Shelftorchlight [36], which displays a projected spotlight (exocentric visual micro guidance) on the target, is beyond the scope of Buchmann et al.'s classification, which focuses on macro guidance techniques and does not take into account the difference between modalities.

Comparative power. The representation of the design space shown in Figure 1 describes techniques in terms of modality, physicality, type, and granularity in a way that helps to identify techniques that are compatible for comparison. To compare two techniques, it is important to have only one parameter that varies. When several parameters vary, it is hard to link the difference in outcomes to a specific parameter.

The first representation classifies techniques in a way that each two horizontally adjacent techniques and techniques in the same column have only one distinct parameter (the modality, type or granularity). As such, for two techniques to be comparable, they should be horizontally adjacent or in the same column.

This representation reveals, for example, that Grohn et al.'s comparison of a 3D audio macro guidance technique to an exocentric visual technique for micro navigation (finding that the audio technique is more effective to orient the user toward the direction of the target compared to the visual condition) [13] varies several parameters simultaneously. It is thus difficult to draw more general conclusions from their results.

This representation, however, only applies to non-hybrid techniques. More specifically, for hybrid techniques, it is necessary to break the individual sub-techniques into their individual components.

Generative power. The generative power of a design space describes the ability to create novel techniques. As before, Figure 1 reveals no guidance system with physical characteristics to support macro navigation. Figure 2 reveals that few guidance techniques apply to both macro and micro search tasks. Furthermore, it provides a suggestion of ways to create new hybrid techniques by blending techniques along the axes into a hybrid technique.

For example, we have used the representation in Figure 2 to identify a new technique, which we call an augmented projection arm, highlighted in yellow in the figure. It combines a physical macro exocentric guidance technique with a micro exocentric visual guidance technique. This prototype is currently under development, and we hope to be able to describe it in future work.

\section{CONCLUSION}

In this work we propose four dimensions to classify guidance techniques: modality, physicality, granularity of the search task and the spatial type of guidance. We then present two complementary representations of the design space. We further discuss its descriptive, comparative and generative power.

Our classification focuses on guidance techniques for the localization of real objects in $3 \mathrm{D}$ real space. It would be interesting, however, to explore the interactions between guidance techniques and localization technology constraints (e.g. precision, momentary failures, blind spots, etc.). 
Our design space is also independent of the technology used to display the information. Future work should take into account the specificities of output devices such as augmented reality glasses.

\section{ACKNOWLEDGMENTS}

This work is funded by Cluster CONNEXION, a French project, and we would like to take this opportunity to thank Catherine Devic, François Cheriaux, Genevieve Filippi, Bernard Nouailhas from EDF and the others collaborators.

\section{REFERENCES}

1. Alastair, D. S., Iain, D. G., and Bruce, M. H. Children's search behavior in large-scale space: Developmental components of exploration. In Journal of Perception, Pion Ltd, 34(10), 2005, pp.1221-1229.

2. Bailey, S. W., Pampel, F. S., Ashmead, D., and Bodenheimer, B. Spatial localization with only auditory cues: a preliminary study. In Proceedings of Applied Perception in Graphics and Visualization '11, ACM, pp. 124-124.

3. Ban, Y., Narumi, T., Tanikawa, T., and Hirose, M. Magic pot: Interactive metamorphosis of the perceived shape. In SIGGRAPH'12, ACM, pp. 1-1.

4. Buchmann, V., Billinghurst, M., and Cockburn, A. Directional interfaces for wearable augmented reality. In SIGCHI New Zealand '08,ACM, pp. 47-54.

5. Butz, A., Schneider, M., and Spassova, M. Searchlight - a lightweight search function for pervasive environments. In PERVASIVE '04, Springer Berlin Heidelberg, pp. 351356.

6. Cholewiak, R. W., and Criag, J. Vibrotactile pattern recognition and discrimination at several body sites. In Journal of Perception \& Psychophysics '84, SpringerVerlag, pp. 503-515.

7. Danieau, F., Lecuyer, A., Guillotel, P., Fleureau, J., Mollet, N., and Christie, M. Enhancing audiovisual experience with haptic feedback: A survey on hav. In HAPTICS '13, IEEE, pp. 193-205.

8. El-Shimy, D., Marentakis, G., and Cooperstock, J. R. Technote: Multimodal feedback in 3d target acquisition. In 3DUI '09, IEEE, pp. 95-98.

9. Ertan, S., Lee, C., Willets, A., Tan, H., and Pentland, A. A wearable haptic navigation guidance system. In International Symposium on Wearable Computers '98, IEEE, pp. 164-165.

10. Gallo, S., Cucu, L., Thevenaz, N., Sengul, A., and Bleuler, $H$. Design and control of a novel thermo-tactile multimodal display. In HAPTICS '14, IEEE, 75-81.

11. Gaver, W. W. Synthesizing auditory icons. In CHI '93, ACM, pp. 228-235.

12. Gonot, A., Chateau, N., and Emerit, M. Usability of 3DSound for Navigation in a Constrained Virtual Environment. In the 120th Audio Engineering Society Convention, Paris, 2006, pp. 1-16.
13. Gröhn, M., Lokki, T., and Takala, T. Comparison of auditory, visual, and audiovisual navigation in a $3 \mathrm{~d}$ space. In Transactions on Applied Perception '05, ACM, pp. 564570 .

14. Guiatni, M., Riboulet, V., Duriez, C., Kheddar, A., and Cotin, S. A combined force and thermal feedback interface for minimally invasive procedures simulation. In Journal of Mechatronics, IEEE/ASME '13, 18(3), pp. 1170-1181.

15. Harada, S., Takagi, H., and Asakawa, C. On the audio representation of radial direction. In $\mathrm{CHI}$ ' $11, \mathrm{ACM}$, pp. 2779-2788.

16. Henderson, S., and Feiner, S. Evaluating the benefits of augmented reality for task localization in maintenance of an armored personnel carrier turret. In ISMAR '09, IEEE, pp. 135-144.

17. Hermann, T., Hunt, A., and John, G. N. The Sonification Handbook. Logos Publishing House, 2011.

18. Hoggan, E., and Brewster, S. Crossmodal spatial location: initial experiments. In NordiCHI '06, ACM, pp. 469-472.

19. Hoshi, T., Takahashi, M., Iwamoto, T., and Shinoda, H. Noncontact tactile display based on radiation pressure of airborne ultrasound. In Haptics '10, IEEE Transaction, pp. $155-165$.

20. Hsia, H. The information processing capacity of modality and channel performance. In Journal of AV communication review'71, 19(1), Springer US, pp. 51-75.

21. Huxtable, B. J., Lai, C. K.-H., Zhu, J. W. J., Lam, P. M.-Y., Choi, Y. T., Neustaedter, C., and Corness, G. J. Ziklo: Bicycle navigation through tactile feedback. In CHI EA '14, ACM, pp. 177-178.

22. Jacobson, R.D. Talking tactile maps and environmental audio beacons: An orientation and mobility development tool for visually impaired people. In Proceedings of the ICA Commission on maps and graphics for blind and visually impaired people' 96, pp. 1-22.

23. Jones, L., and Berris, M. The psychophysics of temperature perception and thermal-interface design. In Jouranl of HAPTICS's '12, IEEE, pp. 137-142.

24. Kahl, G., Spassova, L., Schöning, J., Gehring, S., and Kruüger, A. Irl smartcart - a user-adaptive context-aware interface for shopping assistance. In Proceedings of Intelligent user interfaces '11, ACM, pp. 359-362.

25. Kajimoto, H., Kawakami, N., Maeda, T., and Tachi, S. Tactile feeling display using functional electrical stimulation. In International Conference on Artificial Reality and Telexistence '99, Virtual Reality Soc. of Japan, pp. 259-286.

26. Kientz, J. A., Patel, S. N., Tyebkhan, A. Z., Gane, B., Wiley, J., and Abowd, G. D. Where's my stuff?: design and evaluation of a mobile system for locating lost items for the visually impaired. In Proceedings of Assets '06, ACM, pp. 103-110.

27. Kortum, P. HCI Beyond the GUI: Design for Haptic, Speech, Olfactory, and Other Nontraditional Interfaces. Morgan Kaufmann Publishers Inc., San Francisco, CA, USA, 2008

28. Kruijff, E., Schmalstieg, D., and Beckhaus, S. Using neuromuscular electrical stimulation for pseudo-haptic 
feedback. In Proceedings of Virtual reality software and technology '06, ACM, pp. 316-319.

29. Kuzuoka, H., Oyama, S., Yamazaki, K., Suzuki, K., and Mitsuishi, M. Gestureman: A mobile robot that embodies a remote instructor's actions. In Proceedings of Computer Supported Cooperative Work '00, ACM, pp. 155-162.

30. Larsen, C. H., Lauritsen, D. S., Larsen, J. J., Pilgaard, M., and Madsen, J. B. Differences in human audio localization performance between a hrtf- and a non-hrtf audio system. In Proceedings of Audio Mostly '13, ACM, pp. 1-8.

31. Lecuyer, A., Coquillart, S., Kheddar, A., Richard, P., and Coiffet, P. Pseudo-haptic feedback: Can isometric input devices simulate force feedback. In Proceedings of Virtual Reality '00, IEEE, pp. 83-90.

32. Lehtinen, V., Oulasvirta, A., Salovaara, A., and Nurmi, P. Dynamic tactile guidance for visual search tasks. In UIST '12, ACM, pp. 445-452.

33. Li, M., Arning, K., Bremen, L., Sack, O., Ziefle, M., and Kobbelt, L. Profi: design and evaluation of a product finder in a supermarket scenario. In Proceedings of UbiComp '13, ACM, pp. 977-984.

34. Li, M., Arning, K., Sack, O., Park, J., Kim, M.-H., Ziefle, M., and Kobbelt, L. Evaluation of a mobile projector-based indoor navigation interface. In Journal Interacting with Computers '13, 26(5), pp. 1-19.

35. Lindeman, R. W., and Noma, H. A classification scheme for multi-sensory augmented reality. In Proceedings of Virtual reality software and technology '07, ACM, 175178.

36. Löchtefeld, M., Gehring, S., Schôning, J., and Krüger, A. Shelftorchlight: Augmenting a shelf using a camera projector unit. In Conference on Pervasive Computing ' 10 .

37. Menelas, B., Picinalli, L., Katz, B., and Bourdot, P. Audio haptic feedbacks for an acquisition task in a multi-target context. In Symposium on 3DUI '10, IEEE, pp51-54.

38. Mikawa, M., Morimoto, Y., and Tanaka, K. Guidance method using laser pointer and gestures for librarian robot. In Conference of RO-MAN '10, IEEE, pp. 373-378.

39. Ngo, M., and Spence, C. Auditory, tactile, and multisensory cues facilitate search for dynamic visual stimuli. In Journal of Attention, Perception, and Psychophysics' 10, 72(6), Springer-Verlag, 1654-1665.

40. Nickels, J., Knierim, P., Ko nnings, B., Schaub, F., Wiedersheim, B., Musiol, S., and Weber, M. Find my stuff: Supporting physical objects search with relative positioning. In Proceedings of UbiComp '13, ACM, pp. 325-334.

41. Ogata, K., Seya, Y., Watanabe, K., and Ifukube, T. Effects of visual cues on the complicated search task. In NordiCHI '12, ACM, pp. 478-485.

42. Parseihian, G., and Katz, B. F. G. Morphocons: A new sonification concept based on morphological earcons. In Journal of Audio Eng. Soc'12, 60(6), pp. 409-418.

43. Pfeiffer, M., Schneegass, S., Alt, F., and Rohs, M. Let me grab this: A comparison of ems and vibration for haptic feedback in free-hand interaction. In AH ' 14 , ACM, pp. 48:1-48:8.

44. Prasad, M., Taele, P., Goldberg, D., and Hammond, T. A. Haptimoto: Turn-by-turn haptic route guidance interface for motorcyclists. In CHI '14,ACM, pp. 3597-3606.

45. Pulkki, V. Virtual sound source positioning using vector base amplitude panning. In journal of Audio Eng. Soc '97, 45(6), pp. 456-466.

46. Salzer, Y., Oron-Gilad, T., and Ronen, A. Vibrotactor-belt on the thigh: Directions in the vertical plane. In Proceedings of EuroHaptics '10, Springer Berlin Heidelberg, pp. 359-364.

47. Seco, F., Jimenez, A., Prieto, C., Roa, J., and Koutsou, K. A survey of mathematical methods for indoor localization. In International Symposium on Intelligent Signal Processing' 09, IEEE, pp. 9-14.

48. Simon, R., Jennifer, P., and Jones, M. A billion signposts: Repurposing barcodes for indoor navigation. In CHI '14, ACM, pp. 639-642.

49. Song, Z., Jiang, G., and Huang, C. A survey on indoor positioning technologies. In International Conference Theoretical and Mathematical Foundations of Computer Science '11. Springer Berlin Heidelberg, pp. 198-206.

50. Stahl, C., Baus, J., Brandherm, B., Schmitz, M., and Schwartz, T. Navigational- and shopping assistance on the basis of user interactions in intelligent environments. In The IEE International Workshop on Intelligent Environments '05, IEE, pp. 182-191.

51. Stent, A. J., Azenkot, S., and Stern, B. Iwalk: a lightweight navigation system for low-vision users. In Proceedings of ASSETS '10, ACM, pp. 269-270.

52. Takase, Y., and Hasegawa, S. Presentation of directional information by sound field control. In AH '12, ACM, pp. $32: 1-32: 3$

53. Takeuchi, Y., and Perlin, K. Clayvision: the (elastic) image of the city. In CHI '12, ACM, pp. 2411-2420.

54. Tan, H. Z., Gray, R., Young, J. J., and Traylor, R. A haptic back display for attentional and directional cueing. In Journal of Haptics Research '03, 3(1), pp. 1-20.

55. van Krevelen, D., and Poelman, R. A survey of augmented reality technologies, applications and limitations. In The International Journal of Virtual Reality '10, 9(2), pp. 1-20.

56. Wendy, J., and David, S. Animate objects: How physical motion encourages public interaction. In Proceedings of Persuasive Technology '10, Springer Berlin Heidelberg, pp. 40-51.

57. Yamano, S., Hamajo, T., Takahashi, S., and Higuchi, K. Eyesound: single-modal mobile navigation using directionally annotated music. In AH '12, ACM, pp. 1-4.

58. Yang, G.-H., Kyung, K.-U., Srinivasan, M., and Kwon, D.S. Quantitative tactile display device with pin-array type tactile feedback and thermal feedback. In International Conference on Robotics and Automation '06, IEEE, pp. 3917-3922. 